\title{
DOMAIN AND DOMAIN WALL IMAGES BY SECOND HARMONIC GENERATION
}

\author{
A. Kirilyuk, V. Kirilyuk and Th. Rasing \\ Research Institute for Materials, University of Nijmegen, Toernooiveld 1, 6525 ED Nijmegen, The Netherlands \\ V. V. Pavlov and R. V. Pisarev \\ Ioffe Physical-Technical Institute of the Russian Academy of Sciences, 194021, St. Petersburg, Russia
}

\begin{abstract}
The development of nonlinear optical microscopy based on Magnetization induced Second Harmonic Generation is demonstrated with magnetic garnet films taken as an example. A nontrivial modulated domain structure is shown to exist in a (210) oriented film, along with the possibility to study the magnetization component in the film plane directly and with a strong contrast.
\end{abstract}

KEYWORDS: NONLINEAR MAGNETO-OPTICS, MAGNETIC DOMAINS, GARNET FILMS

\section{INTRODUCTION}

Magnetization induced optical Second Harmonic Generation (MSHG) has been shown to be a very sensitive tool to study magnetic surfaces and interfaces [1-3]. Although the absolute nonlinear signals are small, the magnetization induced effects can be several orders of magnitude larger than their linear counterparts. Recently MSHG has also been used to study the magnetic properties of antiferromagnets. In particular, it gave a unique possibility to visualize the $180^{\circ}$ antiferromagnetic domains in $\mathrm{Cr}_{2} \mathrm{O}_{3}$ single crystals with a non-centrosymmetric magnetic structure [4]. This brings to mind other possibilities, for example, to visualize the magnetic domain pattern at the interface of two different magnetic metals. Also interesting would be to reveal the domain pattern behaviour in thin antiferromagnetic films used as the exchange biasing layer in spin valve structures. These are strong motivations for the further development of MSHG microscopy.

This paper demonstrates the application of MSHG microscopy to the visualization of the domain structures in epitaxially grown magnetic garnet films with thicknesses around $10 \mu \mathrm{m}$. We correlate the observed contrast of the domain structure to the inplane magnetization component, starting from recent studies of MSHG rotational anisotropy. In the (210) oriented films, quite a complicated domain structure exists which is easily made visible with the help of MSHG.

\section{SAMPLES}

Magnetic films were grown by a liquid phase epitaxial method. Gadolinium gallium garnet (GGG) with different crystallographic orientations ((210) or (111)) have been used as a substrate. It appears that the point group symmetry of the magnetic garnet films results from the substrate orientation and growth direction.

As a concequence, inversion symmetry is absent in these films [5]. The point group of the (111) film is $3 \mathrm{~m}\left(\mathrm{C}_{3 \mathrm{v}}\right)$. The only symmetry element for the (210)film is the mirror plane $\mathrm{m}$, which is perpendicular to the film surface. The relevant point group is monoclinic $m\left(\mathrm{C}_{1 \mathrm{~b}}\right)$. In such noncentrosymmetric structures both crystallographic and magnetizationinduced bulk contributions to the nonlinear polarization are allowed at normal and oblique incidences, and thus they can interfere in a magnetized sample or in a sample with a spontaneous magnetic structure. The interference between the nonlinear optical waves leads to a characteristic rotational SHG anisotropy, which has been studied in detail in Ref. [6]. Most of the experiments were done on the (210) film. The spontaneous magnetization in this sample is directed at an angle of $15^{\circ}$ with respect to the film normal. In contrast, the magnetization in the (111) film is exactly perpendicular to the sample surface.

\section{EXPERIMENTAL SETUP}

The SHG signals were measured in transmission geometry at normal incidence (Fig. 1). As a light source we used a Ti:Sapphire laser operating at a repetition rate of $82 \mathrm{MHz}$ and a pulse width of about $100 \mathrm{fs}$. The fundamental beam (with a wavelength of $800 \mathrm{~nm})$ propagates through the films without any noticeable attenuation, but the detected SHG signal comes out only from the back-side layer of about $1 \mu \mathrm{m}$ thickness [7]. The average power on a sample was between 100 and $200 \mathrm{~mW}$. Because of the SHG rotational anisotropy one may probe different directions of magnetisation by changing the incoming light polarisation. A Babinet-Soleil compensator was used to rotate the incoming linear polarization between $0^{\circ}$ and $180^{\circ}$, as well as to create circularly polarized light. The laser beam was focused on the sample into a 


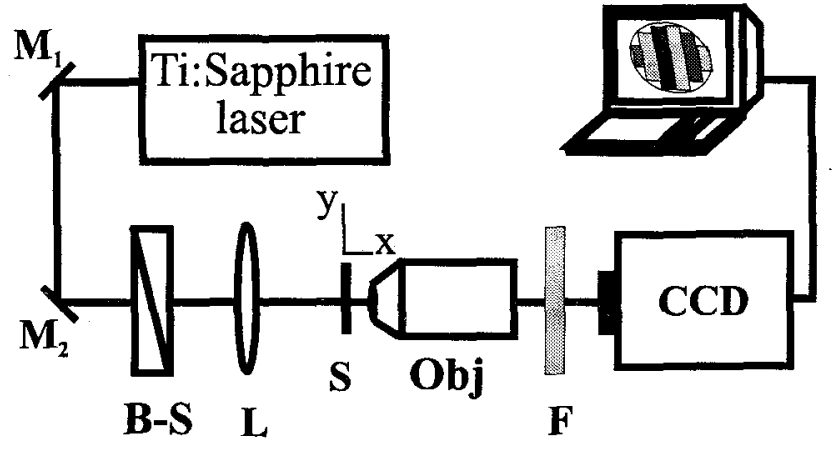

Fig. 1 Experimental setup. $\mathrm{M}_{1}, \mathrm{M}_{2}$ are the mirrors, $\mathrm{B}-\mathrm{S}$ is a Babinet-Soleil compensator, $\mathrm{Obj}$ is a microscope objective, $\mathrm{F}$ is a color filter, $\mathrm{CCD}$ is a liquid nitrogen cooled low noise video camera.

spot of about a $100 \mu \mathrm{m}_{\bar{\lambda}}$ diameter and this exposed area was magnified by a $\times 40$ objective. After appropriate filtering the generated second harmonic intensity was imaged with a cooled CCD camera. The subtraction of the gaussian-like background was applied afterwards to remove the spot-profile induced inhomogeneity of the image intensity.

\section{RESULTS AND DISCUSSION}

First the domain structure of the demagnetized magnetic garnet film (210) was tested with the fundamental light beam. The same experimental setup but with additional analyser was used as a polarising microscope based on the linear Faraday effect. Fig. 2 shows a labyrinth type domain pattern which is quite usual for such type of samples. The width of a single domain was around $10 \mu \mathrm{m}$.

Next, the second harmonic images of the same domain structure were recorded. Due to both the large

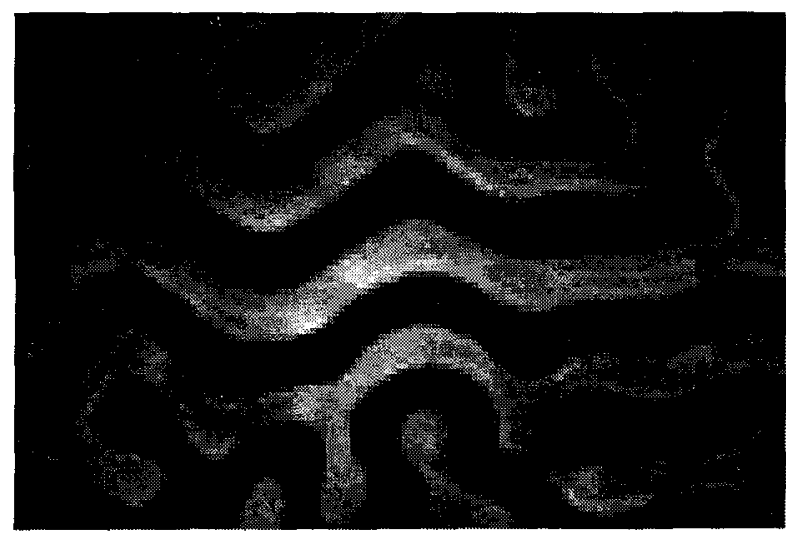

Fig. 2. Magnetic domain structure of a (210) garnet film measured using the Faraday effect for the fundamental beam $(\lambda=800 \mathrm{~nm})$. crystallographic and magnetization-induced bulk contributions to SHG the quality of the images was comparable with those obtained by linear effects.

On the other hand, using the characteristic rotational anisotropy of MSHG found in these films (see Ref. [6]), one can probe different magnetization directions. Indeed, both the second harmonic intensity and the magnetic contrast strongly depend on the mutual directions of the incoming light polarization and the sample magnetization. It is interesting to analyse the different transformations of the domain pattern caused by just changing the incoming light polarization (see Fig. 3).

Fig. 3a demonstrates a domain pattern very similar to that shown in Fig. 2. The incoming light polarization in this case is directed approximately along the $\mathrm{Y}$ axis. If the incoming linear polarisation is rotated now by some $40^{\circ}$ (Fig. $3 \mathrm{~b}$ ), the change of the pattern is clearly observed. To follow all changes appearing in the structure, the domain walls observed in the first image (Fig. 3a) were marked with dashed lines. This also allows to distinguish real shifts from those resulting from small shifts of the incoming fundamental beam due to the polarizing optical components. We see that new domains appear to be shifted by a half of the domain width with respect to those in case a). Of course, this is not a real shift but just a change of contrast which results from the $\mathrm{SH}$ intensity. Why does the MSHG differ in cases a) and b)? In such a configuration, MSHG is sensitive to the in-plane component of the magnetisation [8]. By changing the incoming light polarization different in-plane components are probed. In contrast, only the perpendicular component of the magnetization is measurable with the Faraday effect in the transmition geometry at normal incidence. Therefore we can conclude that in (210) magnetic garnet film every "up" or "down" domain is subdivided into two domains with different in-plane components and these "subdomains" become distinguishable with MSHG. Figs. 3c,d show further evolution of the domain pattern. Rotating the incoming light polarization by an angle of $140^{\circ}$ with respect to the Y-axis (Fig. 3e) an image symmetric (i. e. inverted) with case b) has been recorded. With the linear polarization of the incoming light at $180^{\circ}$ (Fig. 3f), the initial domain pattern has reappeared.

In the (111) film no magnetic domain contrast has been found, although the MSHG was almost of the same order of magnitude as in the (210) film. This can be explained by the fact that the spontaneous magnetization of the domains in the (111) sample is directed normal to the film and no in-plane magnetiza- 


\section{a)}

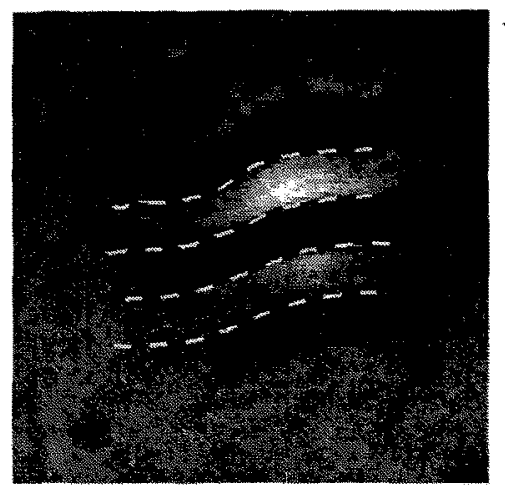

d)

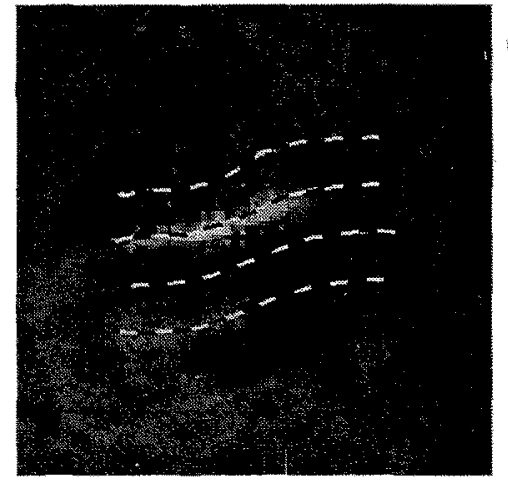

b)

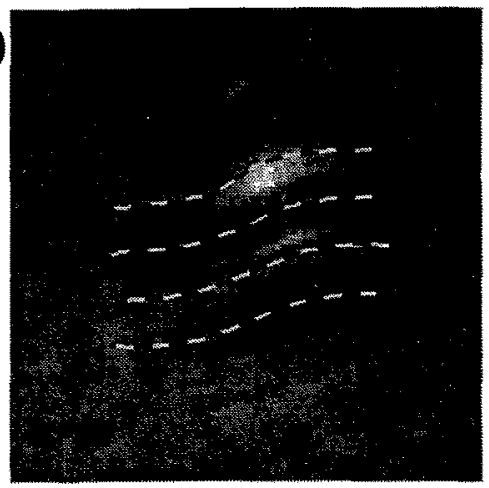

e)

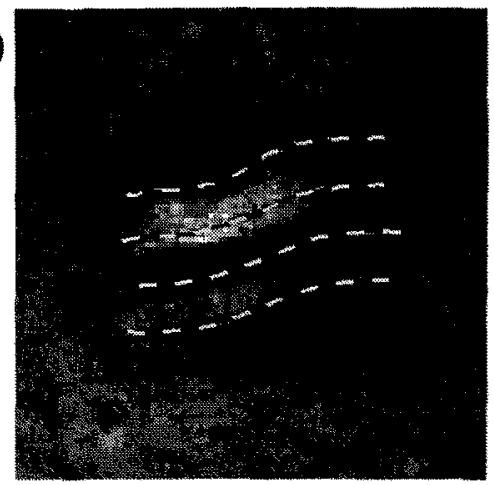

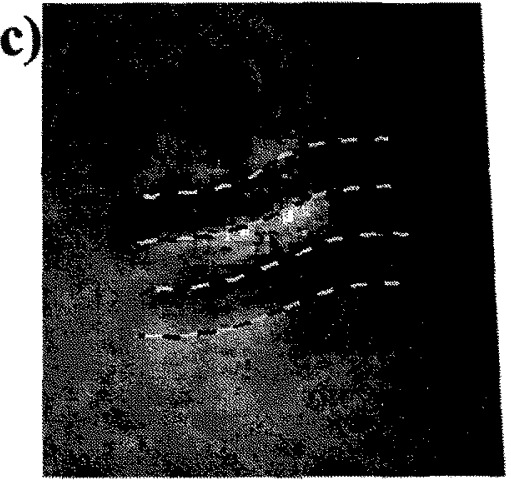

f)

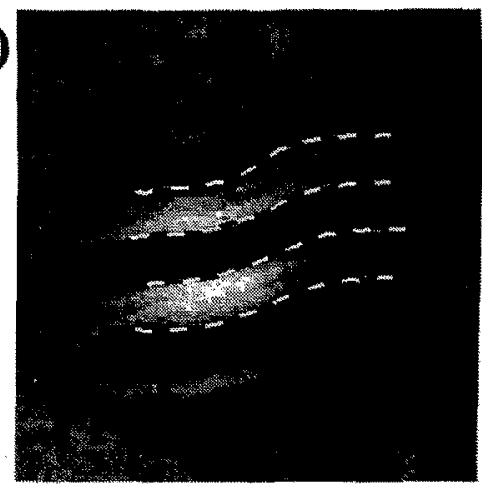

Fig. 3. The domain structure images (the image size is approximately $50 \times 50 \mu \mathrm{m}$ ) obtained with second harmonic light generated in the (210) sample. The direction of the incoming light polarization was a) $0^{\circ}$; b) $40^{\circ}$; c) $100^{\circ}$; d) $120^{\circ}$; e) $140^{\circ}$; f) $180^{\circ}$. Dashed lines show the positions of the visible domains on the first image.

tion component exists.

Recently, MSHG in different garnet films was studied as a function of the mutual direction of the magnetization, light polarization and crystallographic axes (Ref. 6). For this purpose, the magnetization was forced into the film plane with an applied magnetic field. Fig. 4 presents MSHG signals for our (210) film as a function of the rotation angle of the sample.

To make a connection between the curves of Fig. 4 and our domain images, one may consider a simple scheme. Suppose there are two domains ("plus" and "minus") with opposite in-plane magnetization which is directed at some angle $\alpha$ with respect to the crystallographic axis. Let the incoming light polarization be at an angle $\beta$ with respect to the magnetization. It is then obvious that the second harmonic intensity from these two domains can be written as

$$
\begin{aligned}
& J^{+}=I^{+}{ }_{\|}(\alpha) \cos ^{4}(\beta)+I_{\perp}^{+}(\alpha) \sin ^{4}(\beta) \\
& J^{-}=I_{\|}(\alpha) \cos ^{4}(\beta)+I_{\perp}(\alpha) \sin ^{4}(\beta),
\end{aligned}
$$

where $\Gamma^{+,-}$or $\Gamma^{+,-}$denotes the second harmonic intensity for two opposite directions of the magnetization and for the light polarization either parallel or perpendicular to the magnetization. Using the $\mathrm{SH}$ intensities instead of fields, we neglect the SHG with a polarization change which is tested to be small. Taking the values for $\mathrm{I}(\alpha)$ from the curves of Fig. 4, we might be able to make a guess for the value of the angle $\beta$ in the given sample. There are several complications however: (i) the contrast is weaker than calculated from the data of Fig. 4 because the spontaneous inplane component is only a part of the magnetization; (ii) there is still Faraday rotation of the incoming light due to the perpendicular magnetization component. Nevertheless, the data shown here allow us to make a number of plausible conclusions:

1. There are two types of domains which have noncollinear in-plane magnetization components, therefore at least four types of different domains exist;

2. There is no domain type (at least, within the investigated area) with the in-plane magnetization component exactly perpendicular to the mirror plane of the surface.

3. The in-plane components of different non-collinear domains are directed at a rather small angle to each other, say $20-30^{\circ}$. It is also reasonable to suppose that their directions are symmetrical with respect to the mirror plane of our structure. 
a)

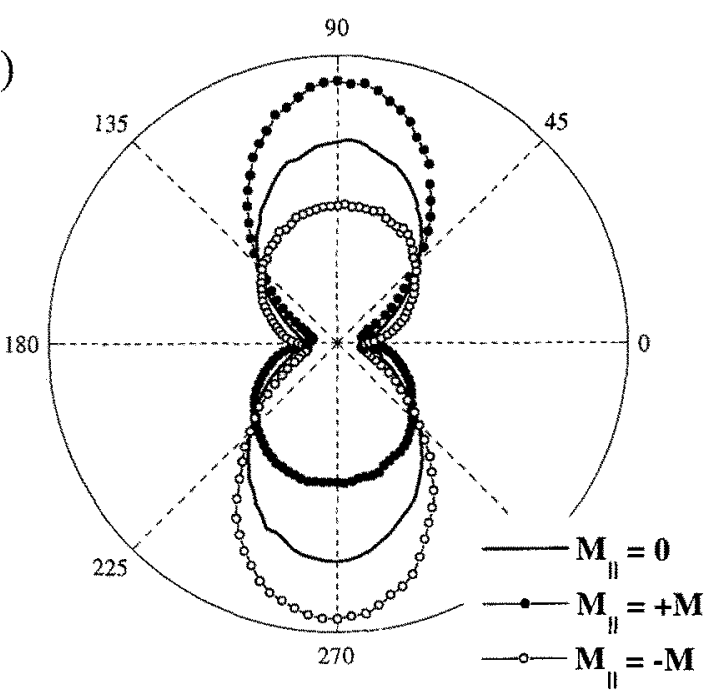

b)

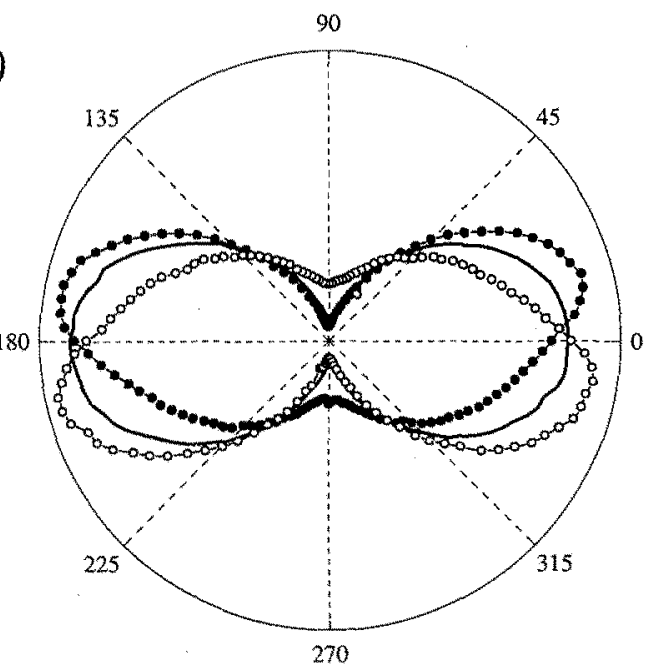

Fig. 4 Second Harmonic intensity as a function of an angle between the crystallographic axes and the incoming light polarization. (a) The direction of the in-plane magnetization $M_{\|}$is fixed perpendicularly to the light polarization $E$; (b) $M_{\|}$is parallel to $E$.

Therefore the determined structure (plus-minus $90^{\circ}$ ) can be summarized as in Fig. 5. With the light polarization nearly perpendicular to the mirror plane, domains $\mathbf{a}$ and $\mathbf{b}$ have different contrast; rotating the polarization we select the domain $\mathbf{c}$ with respect to its neighbours because at some angles the contract between the opposite subdomains in $\mathrm{c}$ is cancelled (see Fig. 4).

We have also found that the MSHG differs at two sides of the magnetic film. In the configuration "film facing the laser", the MSHG signal comes out only from the substrate-film interface (because of the absorption, see Ref. [7]), and in the case "film facing the camera", the MSHG results from the film surface. Almost all experiments have been done with the film facing the laser because larger SH intensity and magne-

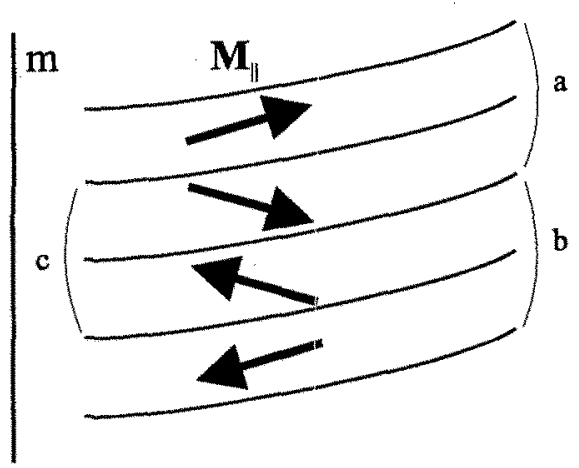

Fig. 5. The domain structure derived from the images. $\mathbf{m}$ is the mirror plane of the film surface.

tic contrast could be achieved with such a configuration.

\section{CONCLUSIONS}

This paper presented a successful development of MSHG microscop tested on magnetic garnet films. Without any polarization analysis, quite complicated domain structure can be resolved. In combination with usual Faraday microscopy (or Kerr, in reflection) it might be a very powerfull tool for domain structure studies.

\section{ACKNOWLEDGEMENTS}

Part of this work was supported by an HCM institutional fellowship ERBCHBGCT930444 and by INTAS-94-2675. We thank A. F. van Etteger for the technical assistance. V.V.P. and R.V.P. acknowledge the hospitality and financial support of the Research Institute for Materials, Nijmegen.

\section{REFERENCES}

[1] J. Reif, J. C. Zink, C. M. Schneider, and J. Kirschner, Phys. Rev. Lett. 67, 2878 (1991)

[2] $\mathrm{H}$. Wierenga, W. de Jong, M. Prins, Th. Rasing, R. Vollmer, A. Kirilyuk, H. Schwabe, and J. Kirschner, Phys. Rev. Lett. 74, 1462 (1995)

[3] B. Koopmans, M. Groot Koerkamp, Th. Rasing, and H. van den Berg, Phys. Rev. Lett. 74, 3692 (1995)

[4] M. Fiebig, D. Frohlich, G. Sluyterman and R. V. Pisarev, Appl. Phys. Lett. 66, 2906 (1995)

[5] B. B. Krichevtsov, V. V. Pavlov and R. V. Pisarev, Sov. Phys. Solid State 31, 1142 (1989)

[6] V. V. Pavlov, R. V. Pisarev, A. Kirilyuk, Th. Rasing, to be published

[7] G. Winkler, Magnetic Garnets (Vieweg, Braunschweig, 1981)

[8] see review by Th. Rasing, these proceedings 